\title{
Existence of the Schmidt decomposition for tripartite systems
}

\author{
Arun Kumar Pati* \\ School of Informatics, Dean Street, University of Wales, Bangor LL 57 1UT, UK.
}

\begin{abstract}
For any bipartite quantum system the Schmidt decomposition allows us to express the state vector in terms of a single sum instead of double sums. We show the existence of the Schmidt decomposition for tripartite system if the partial inner product of a basis (belonging to a Hilbert space of smaller dimension) with the state of the composite system gives a disentangled basis. In this case the reduced density matrix of each of the subsystem has equal spectrum in the Schmidt basis.
\end{abstract}

The key to quantum information processing is the entangled nature of quantum states which has no classical counter part in the theory. These states are central to the study of quantum non-locality, quantum teleportation, quantum cryptography, dense coding and so on [1]. In the simplest term a pure entangled state is a one which cannot be expressed as a direct product of the states of two or more subsystems. If we have a quantum system consisting of two subsystems $A$ and $B$, then the state of the combined system in general can be expressed as $|\psi\rangle_{A B}=\sum_{i j} a_{i j}\left|u_{i}\right\rangle \otimes\left|v_{j}\right\rangle$, where $\left\{\left|u_{i}\right\rangle\right\},\left(i=1,2, \ldots N_{A}\right) \in \mathcal{H}_{A}$ and $\left\{\left|v_{i}\right\rangle\right\},\left(i=1,2, \ldots N_{B}\right) \in \mathcal{H}_{B}$ are the complete set of orthonormal basis vectors in their respective Hilbert spaces. The expansion coefficients in the above state of the combined system contain $N_{A} N_{B}$ terms and is very difficult to manipulate with. However, the Schmidt decomposition (SD) theorem [2] comes to rescue us. It says that any arbitrary state of a bipartite (two-subsystem) quantum system can be expressed as [3]

$$
|\psi\rangle_{A B}=\sum_{i=1}^{N_{A}} \sqrt{p}_{i}\left|x_{i}\right\rangle_{A} \otimes\left|y_{i}\right\rangle_{B}
$$

where $\left\{\left|x_{i}\right\rangle_{A}\right\}$ and $\left\{\left|y_{i}\right\rangle_{B}\right\}$ are two orthonormal basis sets belonging to Hilbert spaces $\mathcal{H}_{A}$ and $\mathcal{H}_{B}$, respectively and $N_{A} \leq N_{B}$. The complex phases can be absorbed in the basis states and the expansion coefficients $\sqrt{p}_{i}$ can be chosen to be real and positive. This simplifies the expression to a great extent.

The Schmidt decomposition theorem has been applied in many worlds interpretation of quantum theory [ []], in reproducing Clebsch-Gordon expansion of angular momentum states

* email:akpati@sees.bangor.ac.uk 
[5], in proving Bell's inequality [6, [] and is quite successful. Recently, in quantum optics context this has been applied [8] and a geometric approach [10] to Schmidt decomposition of two-spin of particles is given in relation to Hardy's proof of quantum non-locality. However, all these discussions pertains strictly to bipartite systems only.

If we have a composite system consisting of more than two subsystems there does not exist a Schmidt decomposition in general. But if it would exist, it will be quite useful simply because the number of terms one deals with in the expansion of the state vector will be comprehensibly small. For example, if we have a quantum system comprising of three subsystems (a tripartite system) then the general state of the system is given by

$$
|\psi\rangle_{A B C}=\sum_{i j k} a_{i j k}\left|u_{i}\right\rangle_{A} \otimes\left|v_{j}\right\rangle_{B} \otimes\left|w_{k}\right\rangle_{C}
$$

where $\left\{\left|u_{i}\right\rangle\right\} \in \mathcal{H}_{A}=C^{N_{A}},\left\{\left|v_{j}\right\rangle\right\} \in \mathcal{H}_{B}=C^{N_{B}}$ and $\left\{\left|w_{k}\right\rangle\right\} \in \mathcal{H}_{C}=C^{N_{C}}$. In this case there are $N_{A} N_{B} N_{C}$ terms to be dealt with. On the other hand if a Schmidt decomposition for tripartite system exists, then we can write the state in (2) as

$$
|\psi\rangle_{A B C}=\sum_{i} \sqrt{p}_{i}\left|x_{i}\right\rangle_{A} \otimes\left|y_{i}\right\rangle_{B} \otimes\left|z_{i}\right\rangle_{C},
$$

where $i=1,2, \ldots N_{A}$ (say) if $N_{A}=\operatorname{dim} \mathcal{H}_{A}$ is the smallest of all the three and $\left\{\left|x_{i}\right\rangle_{A}\right\},\left\{\left|y_{i}\right\rangle_{B}\right\}$ and $\left|w_{i}\right\rangle_{C}$ are again orthonormal basis sets belonging to their respective Hilbert spaces. The general argument which goes against the existence of Schmidt decomposition for a tripartite system such as (3) is that the "equal-spectrum" condition for reduced density matrices does not hold [9]. Nevertheless, it is worth exploring under what conditions SD can exist. If the Schmidt decomposition for a tripartite system exists it would be useful in modal interpretation of quantum theory [1] 13], for example. This possibility has been explored by Peres [14] and he found a necessary and sufficient condition for the existence of a Schmidt decomposition for a tripartite system. However, his condition does not give insight why does it fail for some tripartite systems and why does it always work for a bipartite system. Recently, Thapliyal [15] has discussed Schmidt decomposability for a multipartite systems. He found that if the density matrix is multiseparable after tracing out any party, then the state is Schmidt decomposable.

In this letter we find a simple criterion for the existence of Schmidt decomposition for tripartite system. This gives insight to the question: why does it work always for a bipartite not for a tripartite system. To state it simply, we prove that if the partial inner product of a basis of any one of the subsystems (belonging to a Hilbert space of smaller dimension) with the state of the composite system gives a disentangled basis, then Schmidt decomposition for a tripartite system exists. This condition turns out to be sufficient as well as necessary for the existence of SD. If the partial inner product gives an entangled basis the Schmidt decomposition in terms of a single sum does not exist, though the triple sum can be converted to a double sum. Using our existence criterion we show that the reduced density matrix of each subsystem (by taking partial traces over any two subsystems) has the same eigenvalues, i.e. the "equal-spectrum" requirement holds. Our criterion is also consistent with the existence of Schmidt decomposition for a bipartite system. When we take the partial inner product of any one of the basis with the state of an arbitrary bipartite system, then the resulting basis belong to a Hilbert space of a single subsystem (no question of an entangled basis). This is 
the main reason why the Schmidt decomposition always works for a bipartite system. We also discuss the connection between our criterion and Thapliyal's multiseparability criterion for the existence of SD.

Now we prove the following theorem.

Theorem: For any state $|\psi\rangle_{A B C} \in \mathcal{H}_{A} \otimes \mathcal{H}_{B} \otimes \mathcal{H}_{C}$ of a tripartite system let $\operatorname{dim} \mathcal{H}_{A}=N_{A}$ is smallest of $N_{B}$ and $N_{C}$. If the "partial inner product" of the basis $\left|u_{i}\right\rangle_{A}$ with the state $|\psi\rangle_{A B C}$, i.e. ${ }_{A}\left\langle u_{i} \mid \psi\right\rangle_{A B C}=\left|\psi_{i}\right\rangle_{B C}$ has Schmidt number one then the Schmidt decomposition for a tripartite system exists.

Proof: Let $|\psi\rangle_{A B C}=\sum_{i j k} a_{i j k}\left|u_{i}\right\rangle_{A} \otimes\left|v_{j}\right\rangle_{B} \otimes\left|w_{k}\right\rangle_{C}$ and the partial inner product of the basis $\left|u_{i}\right\rangle$ and state $|\psi\rangle_{A B C}$ is a basis vector in the Hilbert space $\mathcal{H}_{B} \otimes \mathcal{H}_{C}$ spanned by basis vectors $\left\{\left|v_{j}\right\rangle_{B} \otimes\left|w_{k}\right\rangle_{C}\right\}$. It is given by

$$
\left|\psi_{i}\right\rangle_{B C}=\sum_{j k} a_{i j k}\left|v_{j}\right\rangle_{B} \otimes\left|w_{k}\right\rangle_{C}
$$

where $\left\{\left|\psi_{i}\right\rangle_{B C}\right\}$ is an orthogonal basis set but need not be normalised. We know that any vector in a bipartite Hilbert space can be written as a Schmidt decomposition form, i.e.

$$
\left|\psi_{i}\right\rangle_{B C}=\sum_{\mu} \sqrt{p_{\mu}^{(i)}}\left|y_{\mu}\right\rangle_{B} \otimes\left|z_{\mu}\right\rangle_{C}
$$

where $\left\{\left|y_{\mu}\right\rangle_{B}\right\}$ and $\left\{\left|z_{\mu}\right\rangle_{C}\right\}$ are orthonormal basis for $\mathcal{H}_{B}$ and $\mathcal{H}_{C}$ which can be unitarily related to the basis $\left\{\left|v_{j}\right\rangle_{B}\right\}$ and $\left\{\left|w_{k}\right\rangle_{C}\right\}$, respectively. Therefore, the arbitrary state in general can be written as

$$
|\psi\rangle_{A B C}=\sum_{i \mu} \sqrt{p_{\mu}^{(i)}}\left|u_{i}\right\rangle_{A} \otimes\left|y_{\mu}\right\rangle_{B} \otimes\left|z_{\mu}\right\rangle_{C},
$$

Now there can be two situations: (i) either the basis (we call bi-Schmidt basis) $\left\{\left|\psi_{i}\right\rangle_{B C}\right\}$ is entangled or (ii) it is separable. Here, by entangled or separable basis we mean a basis all of whose members are so. We can apply the pure state entanglement criterion, i.e., if the Schmidt number is equal to one then the state is separable and if it is greater than one it is entangled. Here, the Schmidt number is nothing but the number of non-zero eigenvalues in the reduced density matrix of a bipartite system and is the same as the number of terms in the Schmidt decomposition of a bipartite state. This is a good measure of entanglement for pure states. Now imposing this condition on bi-Schmidt basis, we write it as a separable form. So, if $\left|\psi_{i}\right\rangle_{B C}$ has Schmidt number one we can write $\left|\psi_{i}\right\rangle_{B C}=\left|\beta_{i}\right\rangle_{B} \otimes\left|\gamma_{i}\right\rangle_{C}$. Since $\left\{\left|\psi_{i}\right\rangle_{B C}\right\}$ is not normalised $\left\{\left|\beta_{i}\right\rangle_{B}\right\}$ and $\left\{\left|\gamma_{i}\right\rangle_{C}\right\}$ need not be orthonormal though they satisfy orthogonality condition. Therefore, the tripartite system can be written as

$$
|\psi\rangle_{A B C}=\sum_{i}\left|u_{i}\right\rangle_{A} \otimes\left|\beta_{i}\right\rangle_{B} \otimes\left|\gamma_{i}\right\rangle_{C}
$$

Now we calculate the reduced density matrix of each subsystem. The reduced density matrix for $A$ can be obtained by taking partial traces over $B$ and $C$. Thus,

$$
\rho_{A}=\operatorname{tr}_{B}\left(\operatorname{tr}_{C}\left(\rho_{A B C}\right)\right)=\operatorname{tr}_{B}\left[\sum_{i} q_{i}\left|u_{i}\right\rangle_{A A}\left\langle u_{i}|\otimes| \beta_{i}\right\rangle_{B B}\left\langle\beta_{i}\right|\right],
$$


where we have used the trace equality $\operatorname{tr}_{C}\left(\left|\gamma_{i}\right\rangle_{C}\left\langle\gamma_{j}\right|\right)={ }_{C}\left\langle\gamma_{j} \mid \gamma_{i}\right\rangle_{C}=q_{i} \delta_{i j}$ and $q_{i}=\left\|\gamma_{i}\right\|^{2}$ is the (squared) norm of the basis vector $\left|\gamma_{i}\right\rangle_{C}$. Performing the second trace we can write the above one as

$$
\rho_{A}=\sum_{i} q_{i} r_{i}\left|u_{i}\right\rangle_{A A}\left\langle u_{i}\right|
$$

where we have used $\operatorname{tr}_{B}\left(\left|\beta_{i}\right\rangle_{B}\left\langle\beta_{j}\right|\right)={ }_{B}\left\langle\beta_{j} \mid \beta_{i}\right\rangle_{B}=r_{i} \delta_{i j}$ and $r_{i}=\left\|\beta_{i}\right\|^{2}$ is the (squared) norm of the basis vector $\left|\beta_{i}\right\rangle$. Similarly, we can obtain the reduced density matrix $\rho_{B}$ and $\rho_{C}$ as

$$
\begin{aligned}
& \rho_{B}=\sum_{i} q_{i} r_{i}\left|\beta_{i}^{\prime}\right\rangle_{B B}\left\langle\beta_{i}^{\prime}\right| \\
& \rho_{C}=\sum_{i} q_{i} r_{i}\left|\gamma_{i}^{\prime}\right\rangle_{C C}\left\langle\gamma_{i}^{\prime}\right|,
\end{aligned}
$$

where we have defined the orthonormal basis vectors $\left|\beta_{i}^{\prime}\right\rangle_{B}$ and $\left|\gamma_{i}^{\prime}\right\rangle_{C}$ for $\mathcal{H}_{B}$ and $\mathcal{H}_{C}$ as $\left|\beta_{i}\right\rangle_{B}=\sqrt{r}_{i}\left|\beta_{i}^{\prime}\right\rangle_{B}$ and $\left|\gamma_{i}\right\rangle_{C}=\sqrt{q}_{i}\left|\gamma_{i}^{\prime}\right\rangle_{C}$. By comparing all the density matrices, we see that they have same eigenvalue spectrum $\left\{q_{i} r_{i}\right\}$ in the Schmidt basis. Now we can redefine the state of the tripartite system as

$$
\begin{aligned}
|\psi\rangle_{A B C} & =\sum_{i} \sqrt{q_{i} r_{i}}\left|u_{i}\right\rangle_{A} \otimes\left|\beta_{i}^{\prime}\right\rangle_{B} \otimes\left|\gamma_{i}^{\prime}\right\rangle_{C} \\
& =\sum_{i} \sqrt{d}_{i}|i\rangle_{A}|i\rangle_{B}|i\rangle_{C},
\end{aligned}
$$

with $d_{i}=q_{i} r_{i}$ this is the Schmidt decomposition for a tripartite system and hence the proof.

The above proof shows that the disentangled-partial-inner product crietrion is a sufficient one. But it can be checked that it is also a necessary condition, because if SD exists then the partial inner product of a basis in the smallest Hilbert space with the joint state will give a disentangled basis in other two tensor product Hilbert spaces. It should be remarked that $\rho_{A}, \rho_{B}$ and $\rho_{C}$ have the same number of distinct non-zero eigenvalues (non-degenerate spectrum), however, the number of zero-eigen values of $\rho_{A}, \rho_{B}$ and $\rho_{C}$ can be different as $\mathcal{H}_{A}, \mathcal{H}_{B}$ and $\mathcal{H}_{C}$ have different dimensions. The Schmidt decomposition (11) is unique for non-degenerate spectrum of reduced density matrices. The same is true for a bipartite system. Further, when we have a bipartite system then the "partial inner product" of any of the basis with the state of the system gives a single (disentangled) basis for the other one. Hence, the SD is always posible for a bipartite system.

Once we know that the SD exists, then no local unitary operation of the form $U_{B} \otimes$ $U_{C}$, local general measurements and classical communication can disprove the existence of Schmidt decomposition. We know that any measure of entanglement $E\left(\rho_{i, B C}\right)$, with $\rho_{i, B C}=\left|\psi_{i}\right\rangle_{B C B} C\left\langle\psi_{i}\right|$ satisfies the requirements [16] (i) $E\left(\rho_{i, B C}\right)=0$ when $\rho_{i, B C}$ is separable, (ii) $E\left(\rho_{i, B C}\right)=E\left(U_{B} \otimes U_{C} \rho_{i, B C} U_{B}^{\dagger} \otimes U_{C}^{\dagger}\right)$, and (iii) $E\left(\rho_{i, B C}\right)$ cannot increase under local general measurement and classical communication, the Schmidt number of the bi-Schmidt basis does not change and it is impossible to disprove the existence of Schmidt decomposition.

Next we discuss the situation when Schmidt decomposition does not exist for a tripartite system. This is the case when the bi-Schmidt basis is an entangled basis. Physically, this means there exists "entanglement within entanglement". When bi-Schmidt basis is separable there is entanglement between each subsystem $A, B$ and $C$ and there is no " 
entanglement within entanglement". When there is "entanglement within entanglement" the "equal-spectrum" requirement breaks down. However, the "equal-spectrum" holds within the subsystems $B$ and $C$, i.e., $\rho_{B}$ and $\rho_{C}$ have same non-zero eigenvalues. To see this consider the state of a tripartite system as $|\psi\rangle_{A B C}=\sum_{i}\left|u_{i}\right\rangle_{A} \otimes\left|\psi_{i}\right\rangle_{B C}$. The density matrix of the tripartite system is given by

$$
\rho_{A B C}=\sum_{i j}\left|u_{i}\right\rangle_{A A}\left\langle u_{j}|\otimes| \psi_{i}\right\rangle_{B C B C}\left\langle\psi_{j}\right|
$$

On tracing over subsystem $B$ and $C$ we have the reduced density matrix $\rho_{A}$ given by

$$
\rho_{A}=\sum_{i} p_{i}\left|u_{i}\right\rangle_{A A}\left\langle u_{i}\right|
$$

where we have used the trace equality $\operatorname{tr}_{B C}\left(\left|\psi_{i}\right\rangle_{B C B C}\left\langle\psi_{j}\right|\right)={ }_{B C}\left\langle\psi_{j} \mid \psi_{i}\right\rangle_{B C}=p_{i} \delta_{i j}$ and ${ }_{B C}\left\langle\psi_{i} \mid \psi_{i}\right\rangle_{B C}=\sum_{k} p_{k}^{(i)}=p_{i}$ is the (squared) norm of the bi-Schmidt basis. The reduced density matrix $\rho_{A B}$ given by

$$
\rho_{A B}=\operatorname{tr}_{C}\left(\rho_{A B C}\right)=\sum_{i j \mu} \sqrt{p_{\mu}^{(i)} p_{\mu}^{(j)}}\left|u_{i}\right\rangle_{A A}\left\langle u_{j}|\otimes| y_{\mu}\right\rangle_{B B}\left\langle y_{\mu}\right| .
$$

On tracing over the subsystem $A$ we get the reduced density matrix for $\rho_{B}$ given by

$$
\rho_{B}=\sum_{i \mu} p_{\mu}^{(i)}\left|y_{\mu}\right\rangle_{B}\left\langle y_{\mu}\left|=\sum_{\mu} s_{\mu}\right| y_{\mu}\right\rangle_{B B}\left\langle y_{\mu}\right|
$$

where we have defined $\sum_{i} p_{\mu}^{(i)}=s_{\mu}$ and each of them are some positive numbers. To obtain the reduced density matrix for $C$ we first note that $\rho_{A C}$ is given by

$$
\rho_{A C}=\operatorname{tr}_{B}\left(\rho_{A B C}\right)=\sum_{i j \mu} \sqrt{p_{\mu}^{(i)} p_{\mu}^{(j)}}\left|u_{i}\right\rangle_{A A}\left\langle u_{j}|\otimes| z_{\mu}\right\rangle_{C C}\left\langle z_{\mu}\right| .
$$

From the above one we get the reduced density matrix for $\rho_{C}$ given by

$$
\rho_{C}=\sum_{i \mu} p_{\mu}^{(i)}\left|z_{\mu}\right\rangle_{C C}\left\langle z_{\mu}\left|=\sum_{\mu} s_{\mu}\right| z_{\mu}\right\rangle_{C C}\left\langle z_{\mu}\right|
$$

From (15) and (17) these we can see that $\rho_{B}$ and $\rho_{C}$ have same eigenvalue spectrum $\left\{s_{\mu}\right\}$ whereas the eigenvalue of $\rho_{A}$ has different spectrum $\left\{p_{i}\right\}$. Thus, the "equal-spectrum" requirement breaks down when the bi-Schmidt basis $\left\{\left|\psi_{i}\right\rangle_{B C}\right\}$ has Schmidt number greater than one. Interestingly, if we look the subsystems $B$ and $C$ as a single subsystem $B C$, then the "equal-spectrum" requirement holds for subsystems $A$ and $B C$. This is because we have

$$
\rho_{B C}=\sum_{i}\left|\psi_{i}\right\rangle_{B C B C}\left\langle\psi_{i}\right|
$$

On defining an orthonormal basis $\left|\psi_{i}^{\prime}\right\rangle_{B C}$ as $\left|\psi_{i}\right\rangle_{B C}=\sqrt{p}_{i}\left|\psi_{i}^{\prime}\right\rangle_{B C}$, we have

$$
\rho_{B C}=\sum_{i} p_{i}\left|\psi_{i}^{\prime}\right\rangle_{B C B C}\left\langle\psi_{i}^{\prime}\right|
$$


This shows that $\rho_{A}$ and $\rho_{B C}$ have equal-spectrum as expected intuitively.

Before concluding we briefly discuss the link between our criterion and Thapliyal's [15]. We show that the multiseparability criterion discussed in [15 is a special case of our partialinner product criterion when each subsystem has equal dimension. When we have a tripartite system and all the Hilbert spaces have equal dimension, then choosing a Hilbert space of lowest dimension becomes degenerate. Therefore, one has to check if the partial-inner product gives disentangled basis with respect to all the basis vectors and all the parties concerned. For example, if ${ }_{A}\left\langle u_{i} \mid \psi\right\rangle_{A B C}=\left|\psi_{i}\right\rangle_{B C},{ }_{B}\left\langle v_{i} \mid \psi\right\rangle_{A B C}=\left|\phi_{i}\right\rangle_{A C}$ and ${ }_{C}\left\langle w_{i} \mid \psi\right\rangle_{A B C}=$ $\left|\chi_{i}\right\rangle_{A B}$ all gives disentangled basis then there will be a Schmidt decomposition. However, as can be seen if the above conditions hold the density matrices such as $\rho_{B C}, \rho_{A C}$ and $\rho_{A B}$ are multiseparable.

In conclusion, we have found a simple criterion for the existence of Schmidt decomposition for tripartite system and discussed why does it fail in some cases. This also answers why does it always work for a bipartite system. The existence of Schmidt decomposition might be useful in quantifying entanglement content of a pure tripartite system. For example, if the SD exists then the von Neumann entropy of any of the reduced density matrix would give the entanglement content of a pure tripartite system. Our criterion can be generalised to multipartite (more than three) entangled states. Recently, generalised Schimdt-like decomposition has been found for three qubit [17] and multpartite cases [18].

I thank S. L. Braunstein and A. Peres for going through my paper. I also thank C. H. Bennett for useful remarks and interesting questions. The financial support from EPSRC is gratefully acknowledged. 


\section{REFERENCES}

[1] C. H. Bennett and D. P. DiVincenzo, Nature 404, 247 (2000).

[2] E. Schmidt, Math. Ann. 63, 433 (1907).

[3] See for example, A. Peres, Quantum theory:concepts and methods (Kluwer, Dordrecht, $1993)$ p. 123.

[4] H. Everett III, Rev. Mod. Phys. 29, 454 (1957).

[5] S. Bergia, F. Cannata, S. Ruffo, and M. Savoia, Am. J. Phys. 47, 548 (1976).

[6] N. Gisin, Phys. Lett. A 154, 201 (1991).

[7] S. Popescu and D. Rohrlich, Phys. Lett. A 166, 293 (1992).

[8] A. Ekert and P. Knight, Am. J. Phys. 63, 415 (1995).

[9] J. Preskill, Lecture Notes on quantum Information Web page of Preskill.

[10] P. K. Aravind, Am. J. Phys. 64, 1143 (1996).

[11] S.Kochen, "Symposium on the foundations of modern physics" Ed. by P. Lahti and P. Mittelstaedt (World Scientific, Singapore, 1985) P. 151.

[12] E. J. Squires, "Quantum theory without reduction" Ed. by M. Cini and J.M. L'evyLeblond (Hilger, Bristol, 1990) P. 151.J. Phys. A 31, L609 (1998).

[13] R. Healey, Found. Phys. Letts. 6, 37 (1993).

[14] A. Peres, Phys. Lett. A bf 202, 16 (1995).

[15] A. V. Thapliyal, Phys. Rev. A 59, 3336 (1999).

[16] V. Vederal, M. Plenio and P. Knight, Phys. Rev. Lett. 78, 2275 (1997).

[17] A. Acin et al, quant-ph/0003050.

[18] H. A. Carteret, A. Higuchi, A, Sudbry, quant-ph/0006125. 